\title{
Normatizaçãa do eletrorretinograma por reversão alternada de padrões em voluntários normais
}

Standardization of pattern electroretinograms by alternate reversion
in normal volunteers

\author{
Andréa Mara Simões Torigoe ${ }^{1}$ \\ Elizabeth M. A. B. Quagliato ${ }^{2}$ \\ Marcelo Torigoe ${ }^{1}$ \\ Keila Mirian Monteiro de Carvalfio
}

\section{RES UMO}

Objetivo: Realizar a normatização do eletrorretinograma por reversão alternada em indivíduos oftalmologicamente normais e sem doenças neurológicas associadas, determinando a faixa de normalidade estratificada por sexo, faixa etária e estímulo utilizado. Métodos: A padronização seguiu o modelo proposto pela Organização Internacional de Eletrorretinografia e a normatização foi específica para o laboratório de potenciais evocados do Departamento de Neurologia da Faculdade de Ciências Médicas Universidade Estadual de Campinas. Dois tipos de estímulos foram utilizados: o denominado estímulo 16, que proporciona ângulo visual de 60 minutos de arco e o de 32, que proporciona ângulo visual de 30. Resultados: Em todos os pacientes obteve-se uma onda positiva, definida internacionalmente como P50 e uma negativa, chamada N95, sem a presença de artefatos. Foram observados intervalos de normalidade que continham a média das latências, amplitudes e durações das curvas positiva e negativa, internacionalmente aceitas. As ondas P50 e N95 apresentaram diferenças significativas na amplitude, latência e duração quando comparadas às diversas faixas etárias, ocorrendo diminuição na amplitude das ondas e aumento na latência total do eletrorretinograma com o aumento da idade. Construíramse tabelas com intervalo de predição de $95 \%$ em relação à idade para a amplitude, latência e duração das curvas P50 e N95. Conclusões: A normatização do eletrorretinograma por reversão alternada proporciona a reprodutibilidade dos resultados e a possibilidade de estudos comparativos.

Descritores: Eletrorretinografia/normas; Potenciais evocados visuais; Nervo óptico/fisiologia; Reprodutibilidade de resultados

\section{INTRODUÇÃO}

Métodos eletrofisiológicos têm sido desenvolvidos para explorar e definir diferentes estruturas que contribuem para a condução neural da via visual. A atividade bioelétrica das camadas retinianas pode ser avaliada por sinais eletrorretinográficos como respostas a estímulos padronizados ${ }^{(1)}$.

O eletrorretinograma por padrão alternado (PERG) tem possibilitado um avanço na investigação de pacientes com patologias da via visual anterior, permitindo um acesso funcional às células ganglionares da retina. Tem sido descrita, em patologias do nervo óptico, a ausência ou a diminuição do PERG, na presença de ERG normal ${ }^{(2)}$.

O PERG pode ser transiente ou de estado estável, com freqüência temporal baixa equivalente a $3 \mathrm{~Hz}$ ou menos ${ }^{(3)}$. No PERG transiente a onda é caracterizada inicialmente por uma deflexão negativa a $35 \mathrm{~ms}$, que em alguns pacientes é difícil de definir, portanto, utiliza-se a média entre o tempo zero 
e o início do P50. É caracterizado por dois grandes picos: uma onda positiva, com aproximadamente $50 \mathrm{~ms}$ e outra negativa, com aproximadamente $95 \mathrm{~ms}$ (P50 e N95). O componente P50 se encontra alterado nas doenças maculares, já o componente N95 está relacionado às doenças do nervo óptico ${ }^{(4-6)}$.

Prefere-se o estímulo a partir de quadrados ou linhas (verticais ou horizontais), com determinação específica, emitido através de um sistema de televisão, proporcionando imagens seqüenciais a cada 20 ou 16,6 mseg. O padrão deverá ser acromático, branco e preto ${ }^{(7)}$.

Dois tipos de eletrodos são aceitos para registrar o PERG: eletrodos acoplados à lente de contato corneana e eletrodos de contato conjuntival ${ }^{(8)}$. Os de contato conjuntival consistem em eletrodos localizados na pálpebra inferior, em contato direto com a conjuntiva. O protótipo desses eletrodos são os em folhas de ouro que não interferem com a óptica do olho $^{(8)}$, não modificam a refração do paciente e possuem adequada estabilidade elétrica, propiciando uma melhor amplitude de respostas e produzindo menos artefatos que os eletrodos de pele ${ }^{(9)}$.

Ringens et al. em 1984 padronizaram um método para a realização do PERG em seu laboratório, no qual utilizaram eletrodos de folha de ouro colocados internamente na pálpebra inferior (fórnix inferior); o lobo da orelha ipsilateral foi utilizado como referência. $O$ padrão alternado xadrez foi gerado por um monitor de televisão numa freqüência de $2 \mathrm{~Hz}$ e quadrados com $1 \mathrm{grau}^{(10)}$. Observaram que o eletrodo de folha de ouro permitiu a obtenção de ondas com maior amplitude, sendo que a amplitude variou entre 4,0 e $12,1 \mu \mathrm{V}$, com desvio padrão médio de $7,0 \pm 1,6 \mu \mathrm{V}$ e a latência variou entre 44 e $54 \mathrm{~ms}$, com desvio padrão médio de $50 \pm 2 \mathrm{~ms}$, não havendo diferença significativa entre os olhos ${ }^{(10)}$.

Há uma resposta distinta para diferentes laboratórios, ou seja, diferentes respostas obtidas em diferentes locais e épocas, bem como diferentes procedimentos podem resultar em respostas eletrorretinianas equivalentes, resultando em pouca reprodutibilidade dos resultados e perda de informações, sendo necessária a realização de normatizações para cada laboratório de eletrofisiologia ocular ${ }^{(11)}$.

Assim, propôs-se este estudo com o objetivo de normatizar o PERG transiente em indivíduos oftalmologicamente normais e sem doenças neurológicas associadas, determinando a faixa de normalidade estratificada por sexo, faixa etária e estímulo utilizado, especificamente para o laboratório de potenciais evocados do Hospital das Clínicas da UNICAMP.

\section{MÉTODOS}

Foram estudados, prospectivamente, 48 voluntários (96 olhos) (funcionários, pacientes e alunos universitários do $\mathrm{H}$. C. UNICAMP), de acordo com o cálculo do tamanho amostral previamente realizado através de um estudo piloto $^{(12)}$, sem doenças sistêmicas crônicas, sem alterações neurológicas e oftalmologicamente normais (com vício de refração variando de $+3,00$ dioptrias a -3,00 dioptrias, e no máximo 1,50 dioptrias cilíndricas) e acuidade visual 20/20.
Foram separados de acordo com a faixa etária em grupos de 20 a 40 anos (15 pacientes, com média etária de 27 anos e mediana de 29 anos, 8 pertencentes ao sexo feminino), 40 a 60 anos ( 18 pacientes, média de 48 anos e mediana de 50 anos, 10 pertencentes ao sexo feminino) e com mais de 60 anos (15 pacientes, média de 72 anos e mediana de 65 anos, 8 pertencentes ao sexo feminino), definidos como adultos, adultos em idade presbíope e idosos ou senis.

Todos os estudados foram submetidos a exame oftalmológico completo, incluindo medida da pressão intra-ocular (excluídos voluntários com pressão intra-ocular superior a $20 \mathrm{mmHg}$ ), identificados de acordo com o sexo e avaliados unilateralmente, iniciando-se sempre pelo olho direito, com comparação das respostas obtidas.

O protocolo de investigação científica foi aprovado pela Comissão de Ética do Hospital das Clínicas - UNICAMP, e um consentimento informativo foi lido e assinado por cada voluntário submetido ao estudo.

A padronização seguiu o modelo proposto pela International Society of Clinical Eletroretinography, e a normatização foi específica para o laboratório de potenciais evocados situado no Departamento de Neurologia da Faculdade de Ciências Médicas - UNICAMP(13).

Utilizou-se o sistema de medidas de potenciais evocados com amplificação de respostas, MEB-5304K - Nihon Kohden 1992 (Neuropack four-Mini), eletrodos de pele e eletrodos de folha de ouro com contato conjuntival.

A voltagem de calibração variou de $2 \mu \mathrm{V}$ a $1,0 \mathrm{mV}$, com canais variando de $2,5,10,20,50,100,200,500$, e $1000 \mu \mathrm{V}$.

Determinou-se a impedância dos eletrodos de pele, mantendo-a menor ou igual a $5 \mathrm{KOhms.}$

Foram utilizados 3 canais de entrada (registrou-se simultaneamente o potencial visual evocado), sendo que no potencial evocado visual foi empregado um eletrodo ativo na linha média da região occipital e um de referência na linha média da região frontal, próximo à linha de inserção dos cabelos, e no PERG um eletrodo ativo conjuntival de folha de ouro e um de referência no lobo da orelha ipsilateral. Para conseguir um traçado do eletrorretinograma dentro do convencional (positivo para cima), o eletrodo ativo foi ligado à entrada positiva do amplificador. Utilizou-se, também, um eletrodo terra.

A corrente produzida pelo amplificador não ultrapassou $0,01 \mu \mathrm{A}$, e a corrente máxima através dos eletrodos não excedeu $0,5 \mathrm{~mA}$.

Desengordurou-se a pele no local de colocação dos eletrodos com algodão embebido em álcool, sendo os eletrodos de pele fixados com pasta e fita adesiva e o eletrodo conjuntival inserido no terço lateral do fórnix inferior de cada olho e fixado com fita adesiva na pálpebra inferior. Não foram utilizados anestésicos locais.

Foram utilizados, em todos os voluntários, dois tipos de estímulos. O denominado estímulo de 16, que possibilita a visualização no monitor de TV de quadrados com 2,2 centímetros, e o estímulo de 32 , com quadrados de 1,1 centímetros, ambos com a mesma intensidade de luz e contraste em $100 \%$, 
mantendo-se sempre a mesma distância de $100 \mathrm{~cm}$ do olho do paciente à tela do monitor de TV, proporcionando um ângulo visual de 60 minutos de arco nos estímulos de "16", e de 30 minutos de arco nos estímulos de " 32 ".

O estímulo em padrão xadrez alternante foi provido por um sistema de monitor de vídeo VD-401A (17 polegadas, monocromático - 100V 0AC 50/60Hz) com dimensões em 410 (W)x 395(H)x 395(D)mm e freqüência temporal de $2 \mathrm{~Hz}$.

Realizou-se o exame com a melhor correção óptica do paciente para a distância padronizada $(100 \mathrm{~cm})$, em sala clara, com o paciente adaptado à luz durante 5 minutos. Foram amplificados e registrados a amplitude, a latência e o tempo de duração de cada traçado.

Obteve-se a média de 200 apresentações livres de artefatos (traçados não compatíveis com as características das ondas $\mathrm{P}$ e N). O tempo de análise foi de $200 \mathrm{~ms}$.

Metodologia estatística - O número de pacientes necessários para se construir o intervalo de normalidade apresentado foi obtido através do cálculo do tamanho amostral para médias $^{(12)}$.

Calculou-se o mínimo e o máximo, a média e o desvio padrão de cada variável em estudo, estabelecendo-se em seguida uma faixa de normalidade, que foi obtida considerandose a média \pm dois desvios padrões.

Compararam-se as variáveis (amplitude, latência e duração) entre os sexos, idades e estímulos utilizados para cada tipo de onda (N95 e P50), através da análise de variância (ANOVA) com medidas repetidas para experimento fatorial ${ }^{(14)}$. Os fatores utilizados foram o sexo (masculino ou feminino), a idade ( 20 a 40 anos, 41 a 60 anos ou maior que 60 anos) e o estímulo (16 e 32). Os olhos direito e esquerdo foram usados como medida repeti$\mathrm{da}$, devido às medidas serem feitas no mesmo paciente.

A análise de variância foi realizada separadamente para cada variável resposta (amplitude, latência e duração) e para cada onda (N95 e P50).

Caso o efeito de alguma interação fosse estatisticamente significante, far-se-ia a análise controlando-se os efeitos da interação.

Utilizou-se o teste de comparação múltipla de médias de Duncan ${ }^{(13)}$, que compara todos os pares de médias dos níveis do fator desejado.

Utilizou-se o coeficiente de correlação linear de Pearson ${ }^{(14)}$ para verificar a relação entre a variável idade e as variáveis resposta (amplitude, latência e duração).

Para se observar diferenças entre as faixas etárias, realizou-se o teste de comparação múltipla de Bonferroni ${ }^{(14)}$.

Utilizou-se o modelo de regressão linear simples, estimado pelo método dos quadrados mínimos ${ }^{(14)}$ para estabelecer uma equação matemática entre a idade e a variável resposta.

Foi considerado o nível de 5\% de significância, ou seja, $\mathrm{p}<0,05$.

Devido à grande variabilidade das variáveis resposta, foi calculado para cada uma delas o intervalo de predição com $95 \%$ de confiança em função da idade ${ }^{(15)}$.

\section{RESULTADOS}

O tempo total para a realização do exame variou de 15 a 50 minutos, com média de 20 minutos.

Em todos os pacientes obteve-se com facilidade e sem a presença de artefatos uma onda positiva, definida internacionalmente como P50 e uma negativa, chamada N95 (Figura 1).

A duração total da curva foi considerada como a medida do intervalo correspondente ao início da deflexão da onda positiva (P50) à deflexão final da onda negativa (N95).

Determinou-se intervalo normal como a faixa de normalidade de cada variável em estudo.

O intervalo da normalidade médio para a latência da curva P50 foi de 46,53 a $56,15 \mathrm{~ms}$, e para a amplitude da mesma curva 2,60 a $6,89 \mu \mathrm{V}$. Para a curva N95 observou-se um intervalo de normalidade com médias para a latência de 88,19 a $102,95 \mathrm{~ms}$ e para a amplitude 5,45 a $12,50 \mu \mathrm{V}$. A duração total do PERG apresentou o intervalo de normalidade médio de 90,81 a $115,23 \mathrm{~ms}$.

Para os padrões de estímulo 16 e 32, obteve-se em cada variável (latência, amplitude e duração total das curvas P50 e N95), uma faixa de normalidade, considerando a média \pm dois desvios padrões, separando-se o olho direito do esquerdo, a curva, o estímulo e a faixa etária (Tabelas 1a, b e c, respectivamente).

Pela análise da variância para a amplitude da onda N95 (Tabela 2a), verificou-se que apenas o efeito da idade foi significativo ( $p=0,0002)$. É importante notar que não houve diferença significativa de amplitude na onda N95 entre os olhos ou entre os estímulos. Apesar disso, foi feito o teste de comparação múltipla de Duncan para a idade, separadamente para os olhos direito e esquerdo. Em ambos os olhos, a faixa de 20 a 40 anos obteve média de amplitude na onda N95

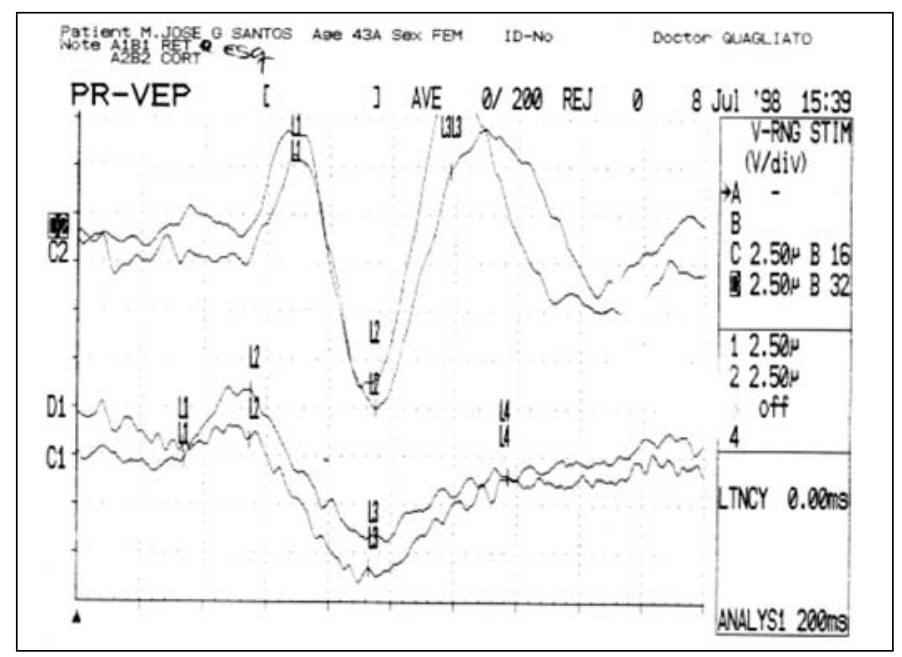

Figura 1 - Registro simultâneo do potencial visual evocado (traçados C2 e D2) e do PERG (traçados C1 e D1) obtido para o olho esquerdo de um paciente do sexo feminino, 43 anos. Realizou-se a repetição de 200 apresentações, tempo de análise $200 \mathrm{~ms}$. 0 ponto marcado com o símbolo L2 representa a onda P50, o símbolo L3 representa a onda N95, ambos nos traçados D1 e C1 


\begin{tabular}{|c|c|c|c|c|c|c|c|c|}
\hline Curva & Estímulo & Idade & $\mathbf{N}$ & $\begin{array}{r}\text { Olho } \\
\text { Média }\end{array}$ & $\begin{array}{l}\text { Direito } \\
\quad \text { Desvio Padrão }\end{array}$ & Máxima & Mediana & Mínima \\
\hline N95 & 16 & $20-40$ & 15 & 9,24 & 1,62 & 11,30 & 9,82 & 5.24 \\
\hline N95 & 16 & $41-60$ & 18 & 8,42 & 1,57 & 10,80 & 8,34 & 4.74 \\
\hline N95 & 16 & $>60$ & 15 & 8,36 & 1,47 & 11,90 & 7,90 & 6.83 \\
\hline N95 & 32 & $20-40$ & 15 & 10,27 & 2,89 & 14,80 & 10,20 & 5.54 \\
\hline N95 & 32 & $41-60$ & 18 & 8,79 & 1,31 & 11,20 & 9,24 & 5.68 \\
\hline N95 & 32 & $>60$ & 15 & 8,52 & 0,98 & 10,00 & 8,51 & 6.80 \\
\hline P50 & 16 & $20-40$ & 15 & 5,65 & 1,21 & 7,34 & 5,39 & 3.55 \\
\hline P50 & 16 & $41-60$ & 18 & 4,19 & 0,93 & 5,68 & 4,29 & 2.10 \\
\hline P50 & 16 & $>60$ & 15 & 4,34 & 0,91 & 6,20 & 4,19 & 2.95 \\
\hline P50 & 32 & $20-40$ & 15 & 5,48 & 1,55 & 7,40 & 5,60 & 2.36 \\
\hline P50 & 32 & $41-60$ & 18 & 4,13 & 1,13 & 5,94 & 3,94 & 2.51 \\
\hline P50 & 32 & $>60$ & 15 & 4,05 & 1,17 & 6,01 & 3,71 & 2.31 \\
\hline \multicolumn{9}{|c|}{ Olho Esquerdo } \\
\hline Curva & Estímulo & Idade & $\mathbf{N}$ & Média & Desvio Padrão & Máxima & Mediana & Mínima \\
\hline N95 & 16 & $20-40$ & 15 & 9,59 & 1,99 & 13,20 & 9,38 & 6.23 \\
\hline N95 & 16 & $41-60$ & 18 & 9,17 & 2,34 & 13,90 & 8,49 & 5.93 \\
\hline N95 & 16 & $>60$ & 15 & 8,30 & 0,73 & 9,28 & 8,53 & 6.94 \\
\hline N95 & 32 & $20-40$ & 15 & 10,73 & 2,94 & 14,60 & 11,80 & 5.24 \\
\hline N95 & 32 & $41-60$ & 18 & 8,56 & 2,34 & 13,00 & 8,00 & 4.32 \\
\hline N95 & 32 & $>60$ & 15 & 7,84 & 0,96 & 9,38 & 8,05 & 5.77 \\
\hline P50 & 16 & $20-40$ & 15 & 5,93 & 1,30 & 7,77 & 6,20 & 3.00 \\
\hline P50 & 16 & $41-60$ & 18 & 4,65 & 1,31 & 6,64 & 4,70 & 2.28 \\
\hline P50 & 16 & $>60$ & 15 & 4,27 & 1,00 & 6,94 & 4,25 & 2.90 \\
\hline P50 & 32 & $20-40$ & 15 & 5,77 & 1,08 & 7,34 & 5,61 & 3.93 \\
\hline P50 & 32 & $41-60$ & 18 & 4,21 & 1,01 & 6,00 & 4,15 & 2.39 \\
\hline P50 & 32 & $>60$ & 15 & 4,20 & 1,04 & 7,47 & 3,96 & 3.20 \\
\hline
\end{tabular}

\begin{tabular}{|c|c|c|c|c|c|c|c|c|}
\hline Curva & Estímulo & Idade & $\mathbf{N}$ & $\begin{array}{l}\text { Olho } \\
\text { Média }\end{array}$ & Desvio Padrão & Máxima & Mediana & Mínima \\
\hline N95 & 16 & $20-40$ & 15 & 95,83 & 3,45 & 101,0 & 95,6 & 89,6 \\
\hline N95 & 16 & $41-60$ & 18 & 95,58 & 3,70 & 103,0 & 96,4 & 88,0 \\
\hline N95 & 16 & $>60$ & 15 & 93,32 & 1,82 & 96,8 & 93,5 & 90,4 \\
\hline N95 & 32 & $20-40$ & 15 & 96,41 & 5,60 & 113,0 & 95,6 & 89,6 \\
\hline N95 & 32 & $41-60$ & 18 & 96,01 & 4,03 & 106,0 & 96,6 & 88,8 \\
\hline N95 & 32 & $>60$ & 15 & 93,15 & 2,07 & 97,6 & 93,6 & 90,4 \\
\hline P50 & 16 & $20-40$ & 15 & 51,09 & 1,68 & 54,8 & 50,4 & 49,6 \\
\hline P50 & 16 & $41-60$ & 18 & 51,24 & 3,36 & 55,8 & 50,8 & 44,8 \\
\hline P50 & 16 & $>60$ & 15 & 51,13 & 1,31 & 52,9 & 51,5 & 48,8 \\
\hline P50 & 32 & $20-40$ & 15 & 51,44 & 2,20 & 57,2 & 50,4 & 49,4 \\
\hline P50 & 32 & $41-60$ & 18 & 51,71 & 3,13 & 57,2 & 50,8 & 46,4 \\
\hline P50 & 32 & $>60$ & 15 & 50,96 & 1,44 & 53,2 & 51,5 & 48,4 \\
\hline \multicolumn{9}{|c|}{ Olho Esquerdo } \\
\hline Curva & Estímulo & Idade & $\mathbf{N}$ & Média & Desvio Padrão & Máxima & Mediana & Mínima \\
\hline N95 & 16 & $20-40$ & 15 & 96,64 & 5,39 & 110,0 & 96,80 & 85,6 \\
\hline N95 & 16 & $41-60$ & 18 & 96,83 & 3,93 & 102,0 & 97,60 & 90,0 \\
\hline N95 & 16 & $>60$ & 15 & 95,21 & 2,50 & 100,0 & 95,21 & 91,6 \\
\hline N95 & 32 & $20-40$ & 15 & 96,98 & 5,13 & 108,0 & 98,00 & 85,2 \\
\hline N95 & 32 & $41-60$ & 18 & 96,64 & 3,97 & 102,0 & 97,20 & 89,6 \\
\hline N95 & 32 & $>60$ & 15 & 94,28 & 2,74 & 100,0 & 94,30 & 90,0 \\
\hline P50 & 16 & $20-40$ & 15 & 50,45 & 2,26 & 54,0 & 50,80 & 44,4 \\
\hline P50 & 16 & $41-60$ & 18 & 51,11 & 2,56 & 55,2 & 51,30 & 46,2 \\
\hline P50 & 16 & $>60$ & 15 & 51,99 & 3,46 & 63,2 & 51,60 & 48,0 \\
\hline P50 & 32 & $20-40$ & 15 & 50,37 & 3,01 & 55,2 & 50,60 & 44,4 \\
\hline P50 & 32 & $41-60$ & 18 & 52,32 & 2,26 & 56,8 & 51,90 & 49,2 \\
\hline P50 & 32 & $>60$ & 15 & 52,32 & 2,18 & 57,2 & 51,97 & 49,8 \\
\hline
\end{tabular}




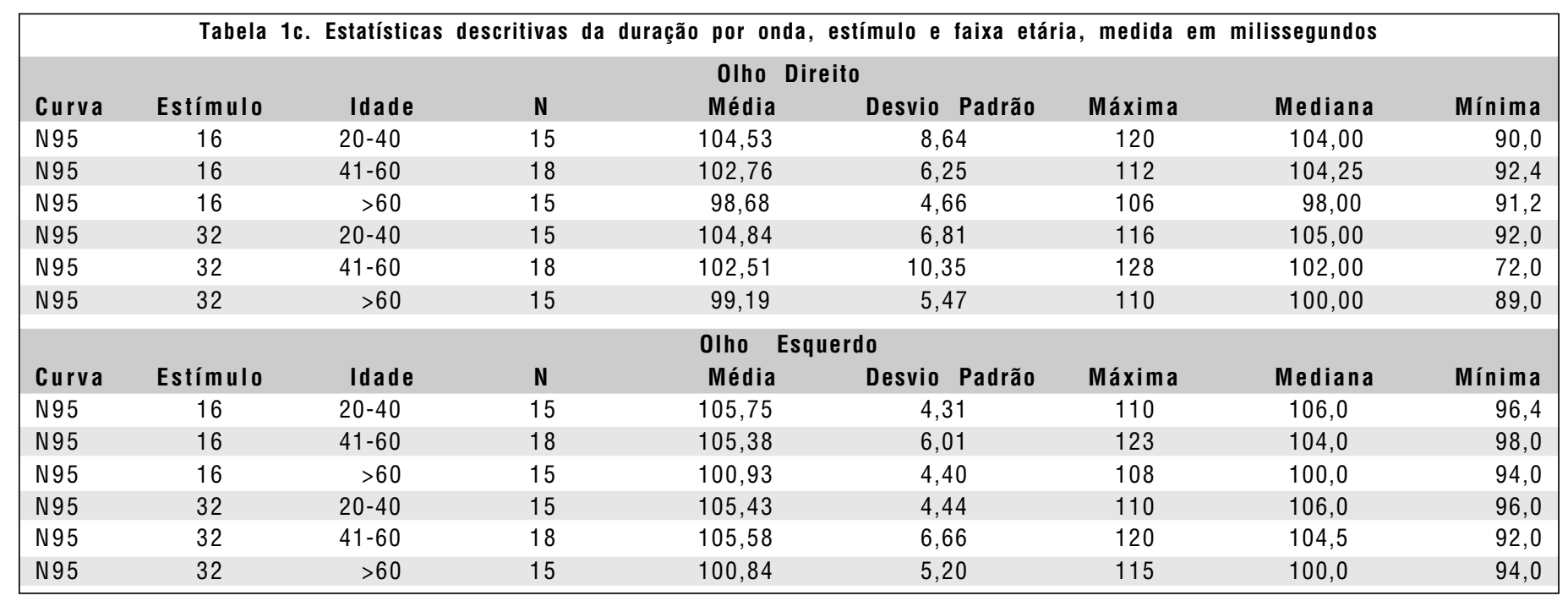

Legendas das tabelas 1a, 1b e 1c - Curva N95: onda negativa do PERG; Curva P50: onda positiva; Estimulo 16: possibilita a visualização no monitor de TV de quadrados com 2,2 cm; estímulo 32 , quadrados de $1,1 \mathrm{~cm}$, com distância constante de $100 \mathrm{~cm}$ do olho do paciente à tela do monitor de TV; N: número de indivíduos em cada amostra

\begin{tabular}{|c|c|c|c|c|c|}
\hline Fonte de variação & Graus de liberdade & Soma de quadrados & Quadrados médios & Estatística & $p$-valor \\
\hline Sexo & 1 & 1,11 & 1,11 & 0,22 & 0,6423 \\
\hline Idade & 2 & 99,57 & 49,78 & 9,69 & 0,0002 \\
\hline Sexo*Idade & 2 & 12,82 & 6,41 & 1,25 & 0,2926 \\
\hline Estímulo & 1 & 1,36 & 1,36 & 0,26 & 0,6083 \\
\hline Sexo*Estímulo & 1 & 3,34 & 3,34 & 0,65 & 0,4220 \\
\hline Idade Estímulo & 2 & 18,41 & 9,20 & 1,79 & 0,1730 \\
\hline Sexo*Idade*Estímulo & 2 & 2,84 & 1,42 & 0,28 & 0,7587 \\
\hline Erro & 84 & 431,78 & 5,14 & & \\
\hline Olho & 1 & 0,22 & 0,22 & 0,10 & 0,7564 \\
\hline Olho*Sexo & 1 & 1,54 & 1,54 & 0,66 & 0,4178 \\
\hline Olho*Idade & 2 & 4,94 & 2,47 & 1,06 & 0,3510 \\
\hline Olho*Sexo*Idade & 2 & 0,18 & 0,09 & 0,04 & 0,9603 \\
\hline Olho*Estímulo & 1 & 2,10 & 2,10 & 0,90 & 0,3449 \\
\hline Olho*Sexo*Estímulo & 1 & 1,21 & 1,21 & 0,52 & 0,4730 \\
\hline Olho*Idade*Estímulo & 2 & 2,01 & 1,00 & 0,43 & 0,6509 \\
\hline Olho*Sexo*Idade Estímulo & 2 & 0,71 & 0,35 & 0,15 & 0,8583 \\
\hline Erro (Olho) & 84 & 196,01 & & & \\
\hline
\end{tabular}

significativamente maior que as outras duas faixas etárias. O mesmo ocorreu para a amplitude da onda P50 (Tabela 2b).

Pela análise de variância para a latência da onda N95 (Tabela 3a), verifica-se que houve diferença significativa entre as idades $(\mathrm{p}=0,0115)$ e entre os olhos ( $\mathrm{p}=0,0306)$, mas não entre os estímulos utilizados. Para a latência da onda P50 (Tabela 3b) não houve nenhum efeito significativo.

Pelo teste de comparação múltipla de Duncan para a idade realizado separadamente para os olhos direito e esquerdo, verifica-se que apenas para o olho direito houve diferença significativa na latência da onda N95 para os voluntários com idade superior a 60 anos, sendo a latência nessa faixa etária, menor que nas outras duas faixas. Para o olho esquerdo, foram estatisticamente iguais.

Para a latência da onda P50 não houve nenhum efeito significativo. Entretanto, para se observar diferenças entre as faixas etárias, realizou-se o teste de comparação múltipla de Bonferroni, separadamente para cada olho, sendo que para o olho direito não foi detectada diferença significativa entre as idades; para o olho esquerdo a média da latência - P50 na faixa etária de 20 a 40 anos foi significativamente menor que para maiores de 60 anos. 


\begin{tabular}{|c|c|c|c|c|c|}
\hline Fonte de variação & Graus de liberdade & Soma de quadrados & Quadrados médios & Estatística $\mathrm{F}$ & p-valor \\
\hline Sexo & 1 & 3,82 & 3,82 & 1,95 & 0,1664 \\
\hline Idade & 2 & 78,65 & 39,32 & 20,05 & 0,0001 \\
\hline Sexo*Idade & 2 & 8,17 & 4,08 & 2,08 & 0,1309 \\
\hline Estímulo & 1 & 2,47 & 2,47 & 1,26 & 0,2648 \\
\hline Sexo*Estímulo & 1 & 0,07 & 0,07 & 0,04 & 0,8470 \\
\hline Idade *Estímulo & 2 & 0,35 & 0,17 & 0,09 & 0,9147 \\
\hline Sexo*Idade Estímulo & 2 & 0,94 & 0,47 & 0,24 & 0,7856 \\
\hline Erro & 84 & 164,73 & & & \\
\hline Olho & 1 & 1,50 & 1,50 & 2,35 & 0,1291 \\
\hline Olho*Sexo & 1 & 0,40 & 0,40 & 0,63 & 0,4279 \\
\hline Olho*Idade & 2 & 0,55 & 0,27 & 0,43 & 0,6494 \\
\hline Olho*Sexo*Idade & 2 & 1,02 & 0,51 & 0,80 & 0,4526 \\
\hline Olho*Estímulo & 1 & 0,33 & 0,33 & 0,52 & 0,4734 \\
\hline Olho*Sexo*Estímulo & 1 & 0,002 & 0,002 & 0,004 & 0,9459 \\
\hline Olho*Idade Estímulo & 2 & 1,84 & 0,92 & 1,44 & 0,2422 \\
\hline Olho* Sexo*Idade ${ }^{*}$ Estímulo & 2 & 3,84 & 1,92 & 3,00 & 0,0549 \\
\hline Erro (Olho) & 84 & 53,76 & 0,64 & & \\
\hline
\end{tabular}

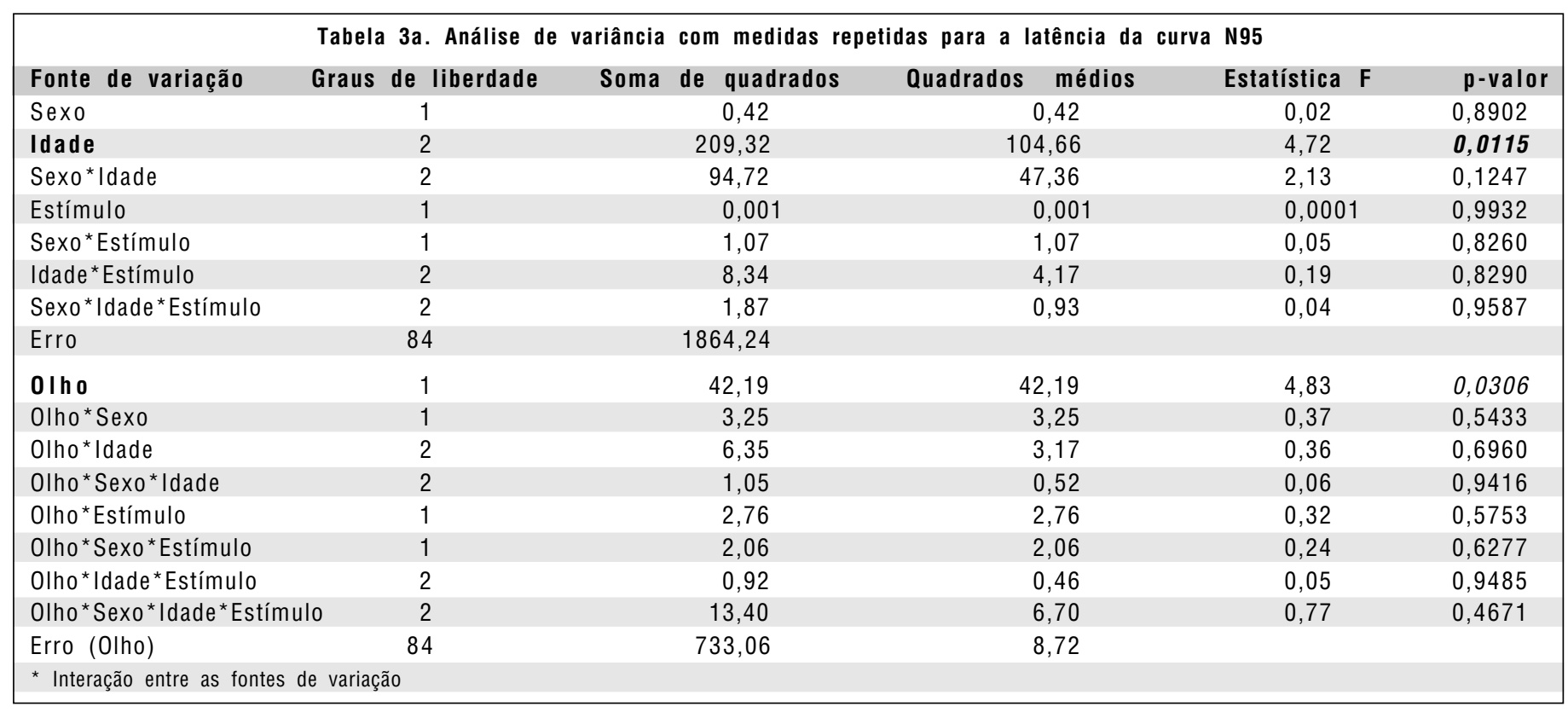

Pela análise de variância para a duração do PERG (Tabela 4) verifica-se que o efeito da interação idade*sexo foi significativo $(p=0,0108)$, bem como o efeito dos olhos $(\mathrm{p}=0,0257)$, mas não o dos estímulos. Assim, fez-se a comparação entre as faixas etárias em cada sexo e após, entre os sexos para cada faixa etária, separadamente por olho.

Fixando-se o sexo feminino, verificou-se uma diferença significativa entre as faixas 41 a 60 anos e maiores de 60 anos no olho direito, e entre maiores de 60 anos e as demais faixas etárias, para o olho esquerdo, pelo teste de comparação múltipla de Duncan, observando-se uma diminuição da duração com o aumento da idade. Para o sexo masculino houve diferen- ça significativa entre a faixa de 20 a 40 anos e as demais, tanto no olho direito como no esquerdo, diminuindo a duração total do PERG com o aumento da idade.

Fixando a faixa etária de 20 a 40 anos, verificou-se diferença significativa entre os sexos apenas para o olho esquerdo, sendo maior a duração para o sexo masculino. Na faixa etária de 41 a 60 anos não houve diferença entre os sexos e para a faixa etária de maiores de 60 anos, houve diferença significativa entre os sexos apenas para o olho esquerdo.

Devido à grande variabilidade das variáveis respostas em função da idade, e aos pequenos valores de $\mathrm{R}^{2}$ obtidos nos modelos de regressão linear (Tabela 5), optou-se pela trans- 


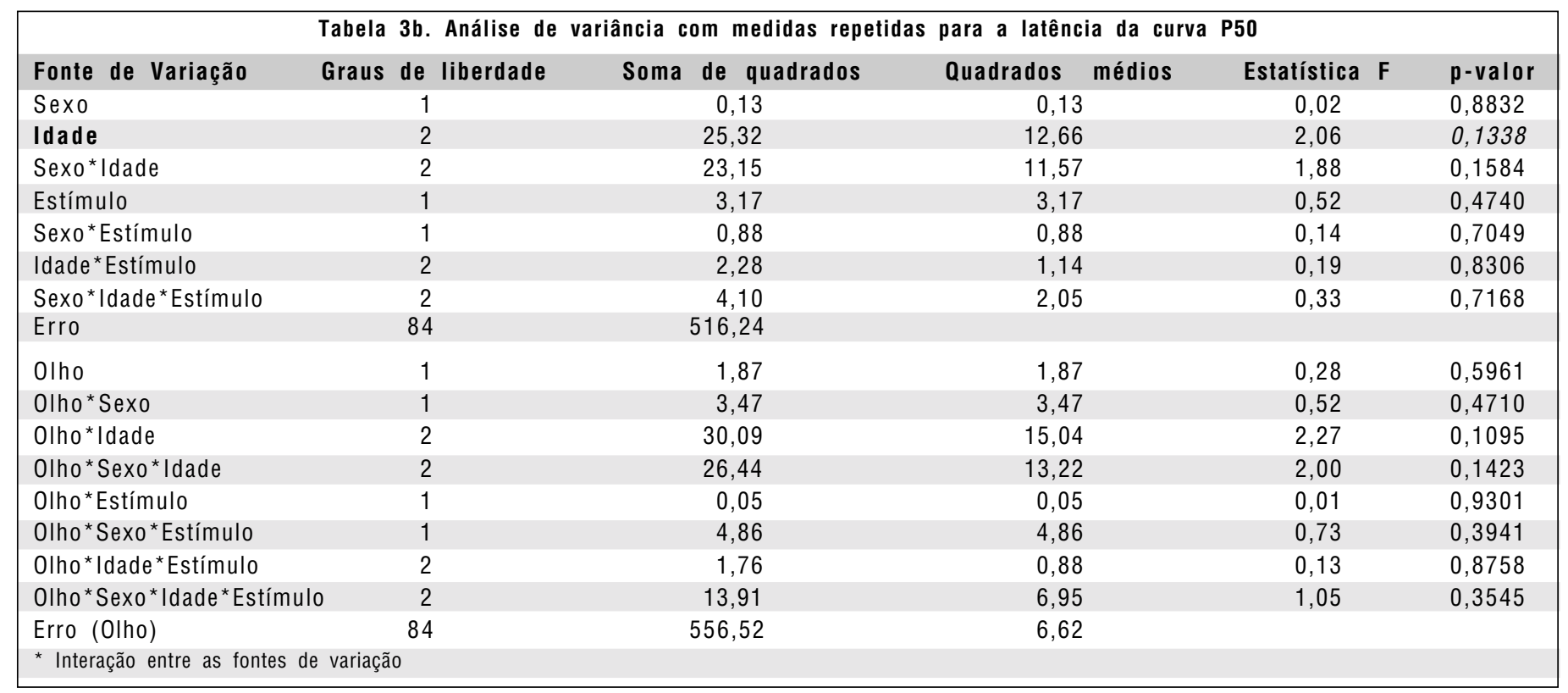

\begin{tabular}{|c|c|c|c|c|c|}
\hline Fonte de Variação & Graus de liberdade & Soma de quadrados & Quadrados médios & Estatística $\mathrm{F}$ & p-valor \\
\hline Sexo & 1 & 77,40 & 77,40 & 1,47 & 0,2281 \\
\hline Idade & 2 & 835,28 & 417,64 & 7,95 & 0,0007 \\
\hline Sexo*Idade & 2 & 501,83 & 250,91 & 4,78 & 0,0108 \\
\hline Estímulo & 1 & 3,48 & 3,48 & 0,07 & 0,7973 \\
\hline Sexo*Estímulo & 1 & 23,20 & 23,20 & 0,44 & 0,5080 \\
\hline Idade Estímulo & 2 & 2,30 & 1,15 & 0,02 & 0,9783 \\
\hline Sexo*Idade*Estímulo & 2 & 12,05 & 6,02 & 0,11 & 0,8917 \\
\hline Erro & 84 & 4411,26 & 52,51 & & \\
\hline OIho & 1 & 146,71 & 146,71 & 5,16 & 0,0257 \\
\hline 01 ho*Sexo & 1 & 3,15 & 3,15 & 0,11 & 0,7397 \\
\hline Olho*Idade & 2 & 23,48 & 11,74 & 0,41 & 0,6630 \\
\hline Olho*Sexo*Idade & 2 & 6,85 & 3,42 & 0,12 & 0,8865 \\
\hline Olho*Estímulo & 1 & 0,14 & 0,14 & 0,01 & 0,9436 \\
\hline Olho*Sexo*Estímulo & 1 & 19,53 & 19,53 & 0,69 & 0,4095 \\
\hline Olho*Idade *Estímulo & 2 & 3,19 & 1,59 & 0,06 & 0,9454 \\
\hline Olho*Sexo*Idade ${ }^{\star}$ Estímulo & 2 & 5,76 & 2,88 & 0,10 & 0.9037 \\
\hline Erro (Olho) & 84 & 2387,83 & 28,42 & & \\
\hline
\end{tabular}

formação das variáveis, usando-se o logaritmo das variáveis resposta em função da idade. Os coeficientes dos modelos de regressão, em sua maioria, continuam significativos, mas os coeficientes de determinação ainda não passam de $19 \%$, mostrando grande dispersão das variáveis resposta.

Essa grande variabilidade motivou o cálculo, para cada uma das variáveis resposta, do intervalo de predição com $95 \%$ de confiança em função da idade ${ }^{(15)}$.

A partir desses dados, construiu-se estatisticamente gráfi$\cos$ (Gráficos 1 a 10) mostrando o intervalo de predição, em relação à idade para cada uma das variáveis. Esses gráficos foram transformados em tabelas que mostram os limites máximo e mínimo dos valores encontrados em 95\% dos indivíduos normais, representando tabelas de normatização do PERG em relação à idade.

\section{DISCUSSÃO}

Em todos os pacientes conseguiu-se com facilidade e sem a presença de artefatos uma onda positiva, definida internacionalmente como P50 e uma negativa chamada N95 (10,16-17).

A média do tempo total para a realização do exame foi de 20 


\begin{tabular}{|c|c|c|c|}
\hline Variável & $\mathbf{R}^{2}$ & Coeficiente & p-valor \\
\hline Amplitude - N95 - OD & 0,1143 & $\begin{array}{l}\text { Interc }=10.87 \\
\text { Idade }=-0.0417\end{array}$ & $\begin{array}{l}0,0001 \\
0,0008\end{array}$ \\
\hline Amplitude - N95 - OE & 0,1639 & $\begin{array}{l}\text { Interc }=11.90 \\
\text { Idade }=-0.0613\end{array}$ & $\begin{array}{l}0,0001 \\
0,0001\end{array}$ \\
\hline Amplitude - P50 - OD & 0,1708 & $\begin{array}{l}\text { Interc }=6.35 \\
\text { Idade }=-0.0370\end{array}$ & $\begin{array}{l}0,0001 \\
0,0001\end{array}$ \\
\hline Amplitude - P50 - OE & 0,2102 & $\begin{array}{l}\text { Interc }=6.76 \\
\text { Idade }=-0.0414\end{array}$ & $\begin{array}{l}0,0001 \\
0,0001\end{array}$ \\
\hline Latência - N95 - OD & 0,0745 & $\begin{array}{l}\text { Interc }=98.45 \\
\text { Idade }=-0.0712\end{array}$ & $\begin{array}{l}0,0001 \\
0,0071\end{array}$ \\
\hline Latência - N95 - OE & 0,0410 & $\begin{array}{l}\text { Interc }=98.80 \\
\text { Idade }=-0.0567\end{array}$ & $\begin{array}{l}0,0001 \\
0,0479\end{array}$ \\
\hline Latência - P50 - OD & 0,0001 & $\begin{array}{l}\text { Interc }=51.32 \\
\text { Idade }=-0.0011\end{array}$ & $\begin{array}{l}0,0001 \\
0,9471\end{array}$ \\
\hline Latência - P50 - OE & 0,0592 & $\begin{array}{l}\text { Interc }=49.32 \\
\text { Idade }=0.0451\end{array}$ & $\begin{array}{l}0,0001 \\
0,0169\end{array}$ \\
\hline Duração - N95 - OD & 0,0977 & $\begin{array}{l}\text { Interc }=109.73 \\
\text { Idade }=-0.1618\end{array}$ & $\begin{array}{l}0,0001 \\
0,0019\end{array}$ \\
\hline Duração - N95 - OE & 0,0797 & $\begin{array}{l}\text { Interc }=109.19 \\
\text { Idade }=-0.1087\end{array}$ & $\begin{array}{l}0,0001 \\
0,0053\end{array}$ \\
\hline \multicolumn{4}{|c|}{$\begin{array}{l}\text { OD - Olho direito; OE - Olho esquerdo; P50 - Curva positiva; N95 Curva } \\
\text { negativa; } \mathrm{R}^{2} \text { - Coeficientes de determinação; Coeficiente - Coeficientes dos } \\
\text { modelos de regressão }\end{array}$} \\
\hline
\end{tabular}

minutos, variando na literatura de 17 a 35 minutos $^{(9)}$, sendo, portanto, um exame de rápida execução.

A utilização do eletrodo de contato conjuntival localizado na pálpebra inferior (eletrodo de folha de ouro) mostrou-se confortável, não interferindo na acuidade visual ou na refração do paciente, e facilmente adaptável, não necessitando do uso de anestésicos oculares, em acordo com a literatura ${ }^{(8-10)}$.

Observaram-se intervalos de normalidade que contêm a média, internacionalmente aceita, da latência e amplitude das curvas positiva e negativa ${ }^{(9-10,17-19)}$.
Comparando-se os grupos especificados quanto às variáveis para as quais as faixas de normalidade foram construídas e considerando diferença significativa valores menores que $5 \%$ (valor de $\mathrm{p}$ ), verificou-se que para a amplitude das curvas P50 e N95 o efeito idade foi o mais significativo $(p=0,0002)$, ocorrendo diminuição da amplitude com o aumento da idade, mostrando-se de acordo com a literatura ${ }^{(10,20-22)}$.

Para a latência das curvas houve diferenças significativas entre as faixas etárias e entre os olhos analisados (direito ou esquerdo), ocorrendo uma diminuição na latência da curva N95 para os pacientes acima de 60 anos, somente para a análise do olho direito (todos os exames foram iniciados pelo olho direito) e houve também um aumento da latência para a curva P50 para os indivíduos acima de 60 anos. Assim, observou-se um aumento na latência total do PERG com o aumento da idade, como sugere a literatura ${ }^{(10,20-22)}$.

Analisando a duração total do PERG, verificou-se um efeito da interação idade e sexo, diminuindo a duração com o aumento da idade, principalmente acima de 60 anos para o sexo feminino e acima de 40 anos para o sexo masculino. $O$ fator sexo, isoladamente, não determinou diferenças significativas entre as variáveis, como sugere a literatura ${ }^{(2,23-25)}$, porém não encontramos na literatura análise interativa entre sexo e idade.

Analisando os resultados comparados entre os padrões de estímulos de 16 e 32, estratificados nas faixas etárias, constatou-se que não houve diferenças significativas entre as variáveis (latência, amplitude e duração) nas curvas P50 e N95. Portanto, nesses indivíduos oftalmologicamente normais, o tamanho do quadrado do estímulo apresentado não interferiu na função foveal ou no campo de observação, como refere à literatura $^{(9-10)}$

Sendo muitas as variáveis que influenciam o eletrorretinograma, não se deve comparar os valores obtidos em um laboratório com outros ${ }^{(25-27)}$. Apesar disso, os nossos resultados foram semelhantes aos encontrados na literatura ${ }^{(9-10,17,19)}$.
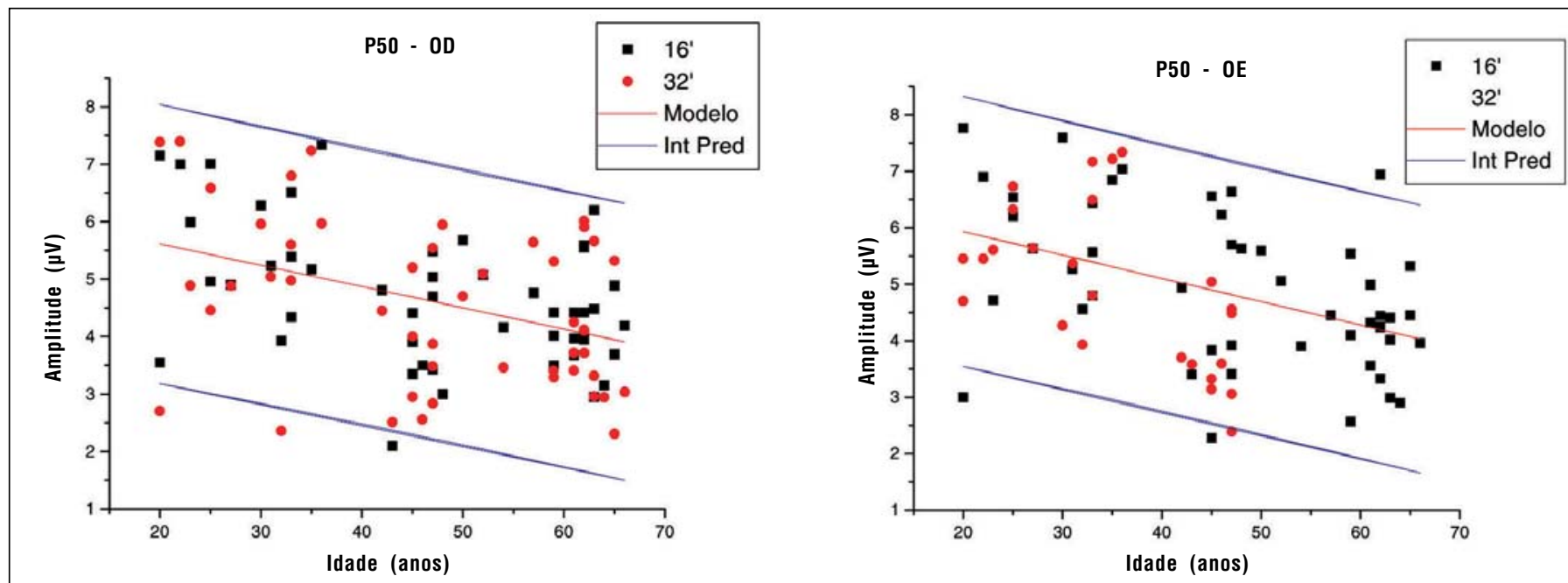

Gráficos 1a e 1b - Relação entre amplitude para a curva P50 e idade para olhos direito e esquerdo 


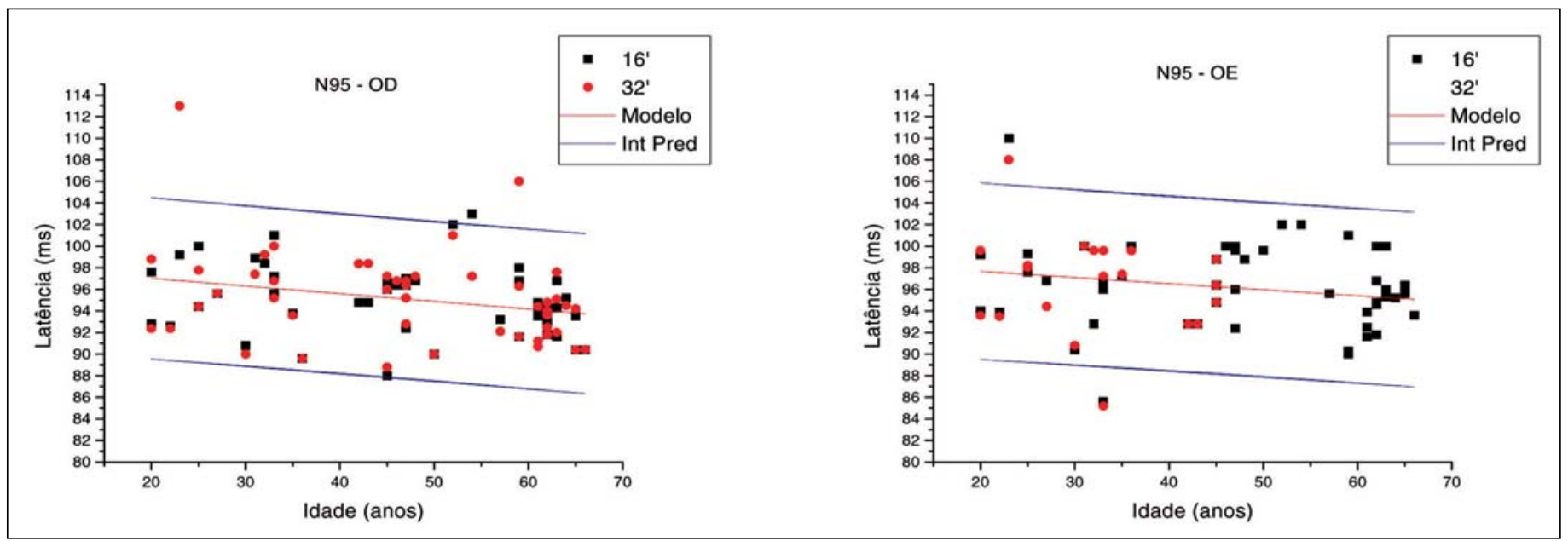

Gráficos 2a e 2b - Relação entre latência para a curva N95 e idade para olhos direito e esquerdo

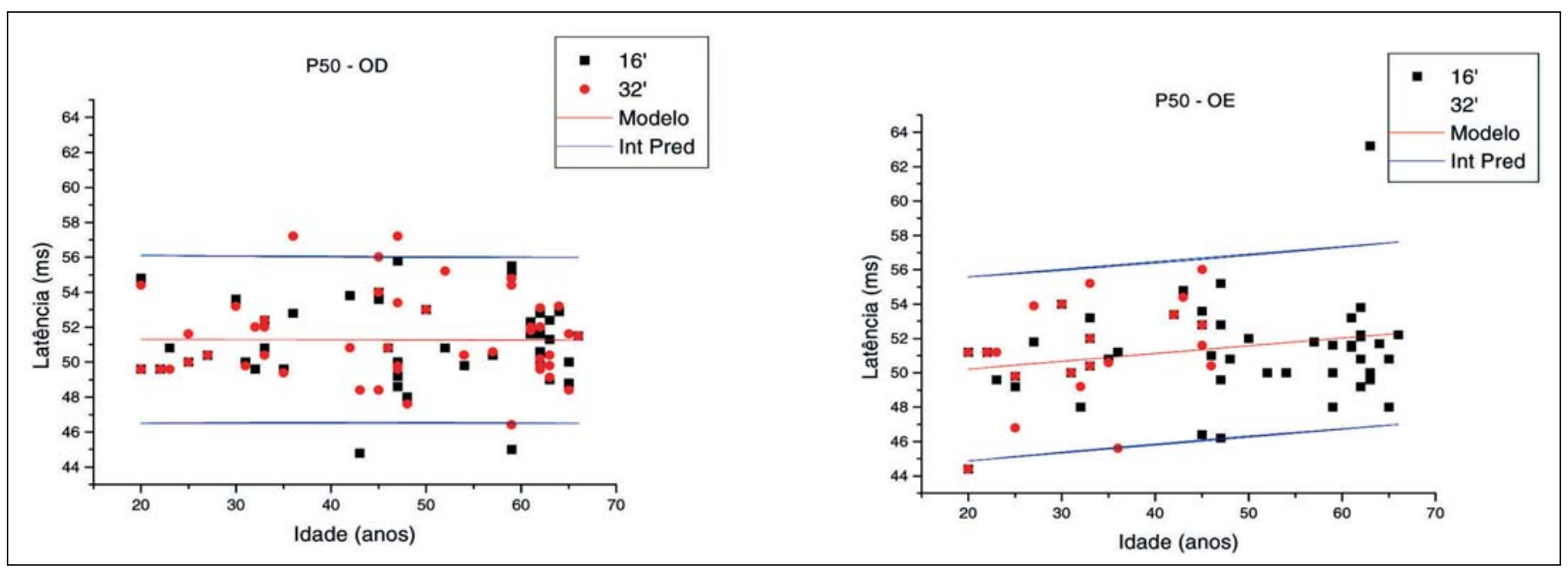

Gráficos 3a e 3b - Relação entre latência para a curva P50 e idade para olhos direito e esquerdo

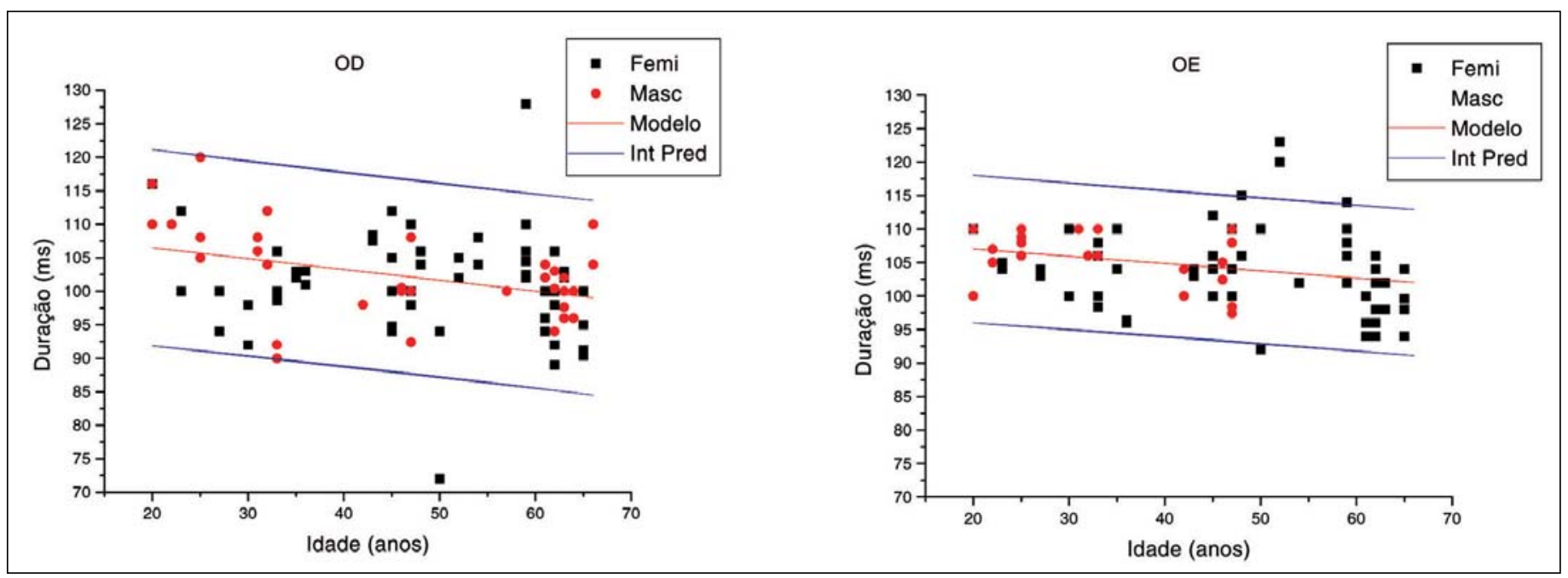

Gráficos 4a e 4b - Relação entre duração do PERG e idade para olhos direito e esquerdo 


\section{CONCLUSÃO}

$\mathrm{Na}$ avaliação do PERG em indivíduos oftalmologicamente normais e sem doenças neurológicas associadas, estabeleceu-se a faixa de normalidade para a curva positiva (P50), com latências variando de 46,53 a $56,53 \mathrm{~ms}$ e amplitudes variando de 2,60 a $6,89 \mu \mathrm{V}$. Obteve-se para a curva negativa (N95) latências variando de 88,19 a $102,95 \mathrm{~ms}$ e amplitudes variando de 5,45 a $12,50 \mu \mathrm{V}$. A duração total das curvas variou de 90,81 a $115,23 \mathrm{~ms}$.

A normatização do eletrorretinograma por reversão alternada proporciona a reprodutibilidade dos resultados e a possibilidade de estudos comparativos.

\section{ABS TRACT}

Purpose: To standardize recordings of pattern electroretinograms (PERG) in normal human subjects. Methods: The standardization followed the model proposed by the International Organization of Eletroretinography and was specific for the laboratory of evoked potentials of the Department of Neurology, Faculty of Medical Sciences, State University of Campinas. Two types of stimuli were used, one of 30 minutes of arch of visual angle and another of 60 minutes of arch of visual angle. Results: In all patients a positive wave, defined internationally as P50, and a negative, called N95, were obtained without interferences. The normal range for latency, amplitude and duration of each wave was obtained. There were significative differences in amplitude, latency and duration of P50 and N95 waves among the several age groups. There was an increase in latency and a decrease in amplitude with increasing age. No significative differences were found among the variables comparing different stimuli, sex or when eyes were compared with each other. Conclusions: The standardization of pattern electroretinograms yield reproductibility of results and the possibility of further comparative studies.

Keywords: Electroretinography/standards; Evoked potentials, visual; Optic nerve/physiology; Reproducibility of results

\section{REFERENCIAS}

1. Trimarchi C, Biral G, Domenici L, Porciatti V, Bisti S. The flash and pattern electroretinogram generators in the cat: a pharmacological approach. Clin Vis Sci 1990;6:19-24.

2. Dawson WW, Maida TM, Rubin ML. Human pattern-evoked retinal responses are altered by optic atrophy. Invest Ophthalmol Vis Sci 1982;22:796-803.

3. Bach M, Hawlina M, Holder GE, Marmor MF, Meigen T, Vaegan, Miyake Y. Standard for pattern electroretinography. Doc Ophthalmol 2000;101:11-8.
4. Fiorentini A, Maffei L, Pirchio M, Spinelli D, Porciatti V. The ERG in response to alternating gratings in patients with diseases of the peripheral visual pathway. Invest Ophthalmol Vis Sci 1981;21:490-3.

5. Ringens PJ, Vijfvinkel-Bruinenga S, Van Lith GH. The Pattern-elicited electroretinogram. I. A tool in the early detection of glaucoma? Ophthalmologica 1986;192:171-5.

6. Mendonça RHF, Takahashi WY, Usuba FS, Mendonça CHF. Alterações eletrorretinográficas na distrofia de cones. Rev Bras Oftalmol 1999;58:21-5.

7. Celesia GG, Bodis-Wollner I, Chatrian GE, Harding GF, Sokol S, Spekreijse $\mathrm{H}$. Recommended standards for electroretinograms and visual evoked potentials. Report of na IFCN Committee. Electroencephalogr Clin Neurophysiol 1993;87:421-36.

8. Arden GB, Carter RM, Hogg C, Siegel LM, Margolis S. A gold foil electrode: extending the horizons for clinical electroretinography. Invest Ophthal Vis Sci 1979;18:421-6.

9. Tan CB, King PJ, Chiappa KH. Pattern ERG: effects of reference electrode site, stimulus mode and check size. Electroencephalogr Clin Neurophysiol 1989;74:11-8

10. Ringens PJ, Van Lith GH, Vijfvinkel-Bruinenga S. A standardized method for recording pattern-elicited electroretinograms in normal human subjects. Ophthalmologica 1984;188:106-10.

11. Van Der Tweel LH, Carr R, Hellner KA, Lawwill T, Van Lith GH, Tazawa Y. Report of the committee on instrumentation and procedures in visual electrophysiology at the request of the Concilium Ophthalmologicum Universale. Doc Ophthalmol 1981;51:383-95.

12. Fonseca JS, Martins GA. Curso de Estatística, 5ª ed. São Paulo: Atlas; 1994

13. ISCERG - International Society of Clinical Eletroretinography. Stockholm 1961. Acta Ophthalm 1962;(Suppl.70):264-8.

14. Montgomery DC. Design and analysis of experiments. New York: John Wiley \& Sons; 1991.

15. Montgomery DC, Peck EA. Introduction to linear regression analysis. New York: John Wiley \& Sons; 1982.

16. Parisi V. Neural conduction in the visual pathways in ocular hypertension and glaucoma. Graefes Arch Clin Exp Ophthalmol 1997;235:136-42.

17. Parisi V, Uccioli L, Parisi L, Colacino G, Manni G, Menzinger G et al. Neural conduction in visual pathways in newly-diagnosed IDDM patients. Electroencephalogr Clin Neurophysiol 1998;108:490-6.

18. Babel J, Stangos N, Korol S, Spiritus M. Ocular electrophysiology: a clinical and experimental study of electroretinogram. eletro-oculogram, visual evoked response. Stutgart: Thieme; 1977.

19. Korth M. Pattern-evoked responses and luminance-evoked responses in the human electroretinogram. J Physiol 1983;337:451-69.

20. Wright CE, Williams DE, Drasdo M, Harding GF. The influences of age in the electroretinogram and visual evoked potential. Doc Ophthalmol 1985;59: 365-84.

21. Török B, Meyer M, Wildberger H. The influence of pattern size on amplitude, latency and wave form of retinal and cortical potentials elicited by checkerboard pattern reversal and stimulus onset-offset. Electroencephalogr Clin Neurophysiol 1992;84:13-9.

22. Celesia GG, Kaufmann D, Cone SB. Simultaneous recording of pattern electroretinography and visual evoked potentials in multiple sclerosis. A method to separate demyelination from axonal damage to the optic nerve. Arch Neurol 1986;43:1247-52.

23. Gonçalves E. Eletrorretinografia. In: XVI Congresso Brasileiro de Oftalmologia 1971; Campinas, São Paulo; 1971. Anais. p.33-45.

24. Sokol LL, Riggs LA. Electrical and psycophysical responses of the human visual system to periodic variation of luminance. Invest Ophthal 1971;10: 171-80.

25. Pacini L. Eletrorretinografia-ERG. Rev Bras Oftalmol 1979;33:147-72.

26. Costa RHM, Takahashi WY, Dias NT. Normatização do eletrorretinograma. Rev Bras Oftalmol 1998;57:375-8.

27. Paranhos FRL, Ávila MP, Paranhos A, Cialdini AP. Estudo estatístico de valores normais do eletrorretinograma. Contribuição à padronização do exame. Arq Bras Oftalmol 1997;60:278-84. 